\title{
Curva crítica de diluição do nitrogênio para a cultura do melão
}

\author{
Nitrogen critical dilution curve for the muskmelon crop
}

\begin{abstract}
Marco Aurélio de Freitas Fogaça' Jerônimo Luiz Andriolo*II Rodrigo dos Santos Godoi ${ }^{\text {III }}$ Cláudia Alessandra Peixoto de Barros ${ }^{\text {III }}$ Djeimi Isabel Janisch ${ }^{\text {III }}$ Marcos André Braz Vaz ${ }^{\text {II }}$
\end{abstract}

\section{RESUMO}

O objetivo do trabalho foi ajustar a curva crítica de diluição do nitrogênio da cultura do melão. O experimento foi conduzido em ambiente protegido na Universidade Federal de Santa Maria (UFSM), entre agosto de 2004 e janeiro de 2005. As mudas do híbrido Magellan foram plantadas em sacolas de polietileno contendo $4,5 \mathrm{dm}^{-3}$ de substrato comercial (Plantmax $P X T^{\circledR}$ ), na densidade de 3,3 plantas $\mathrm{m}^{-2}$ e fertirrigadas com solução nutritiva completa. As plantas foram conduzidas verticalmente com uma haste, deixando-se no máximo dois frutos por planta e foram podadas ao atingir a altura de $2 \mathrm{~m}$. Os tratamentos foram constituídos por concentrações de nitrogênio na solução nutritiva de 8; 11, 14; 17 e $20 \mathrm{mmol} \mathrm{L}^{-1}$. O delineamento experimental utilizado foi o inteiramente casualizado com quatro repetições. Quatro plantas de cada tratamento foram coletadas semanalmente entre os 33 e 99 dias após o plantio para determinar o acúmulo de matéria seca (MS) e o teor de $N$ nas folhas, haste e frutos. Foi constatada a diluição da concentração de $N$ na matéria seca em todos os tratamentos e os dados ajustaram-se ao modelo potencial $\% N=a M S^{-b}$ descrito na literatura. A curva crítica de diluição do $N$ foi ajustada, com coeficientes a e b iguais a 5,16 e 0,63, respectivamente. Esse modelo poderá ser usado para estimar a quantidade de $N$ extraída no decorrer do ciclo de crescimento e desenvolvimento dessa cultura, com base na análise do teor desse nutriente nas folhas.

Palavras-chave: Cucumis melo, alometria, adubação, matéria seca.

\section{ABSTRACT}

The research was carried out to adjust the nitrogen critical dilution curve for the muskmelon crop, to be used in fertilization practices for this crop. The experiment was conducted in a greenhouse at Universidade Federal de Santa
Maria, from August to January, 2005. Plantlets of the hybrid Magellan was grown in polyethylene bags with $4.5 \mathrm{dm}^{3}$ of the commercial substrate Plantmax $P X T^{\circledR}$, in a plant density of 3.3plants $\mathrm{m}^{-2}$, and fertigated with a complete nutrient solution. Plants were vertically trained with one stem and no more than two fruits per plant, and the main stem was cut at $2 \mathrm{~m}$ height. Treatments were $N$ concentrations in the nutrient solution of 8 , $11,14,17$, and $20 \mathrm{mmol} \mathrm{L}^{-1}$, in a randomized experimental design with four replications. Four plants of each treatment were harvested at weekly intervals between 33 and 99 days after planting to determine dry mass (DM) accumulation and $N$ concentration in leaves, stem and fruits. The $N$ dilution in plant dry mass was confirmed in all treatments and data fitted the potential model $\% N=a M S^{-b}$ described in the literature. The $N$ dilution curve was adjusted, with values of 5.16 and 0.63 for $a$ and $b$ coefficients, respectively. This model could be used to estimate the $N$ quantity absorbed during growth and development of this crop, based on the analysis of this element on leaf tissues.

Key words: Cucumis melo, alometrie, fertilization, dry mass.

\section{INTRODUÇÃO}

O nitrogênio (N) é o nutriente envolvido diretamente com o crescimento vegetativo das plantas, especialmente em relação à área foliar. Relações lineares têm sido demonstradas na literatura entre a taxa de crescimento das culturas e a concentração desse elemento na planta, as quais constituem a base teórica para os conceitos sobre a eficiência de uso do N (INGESTAD \& ÅGREN, 1992; VERKROOST \&

IPrograma de Pós-graduação em Agronomia (PPGA), Universidade Federal de Santa Maria (UFSM), Santa Maria, RS, Brasil.

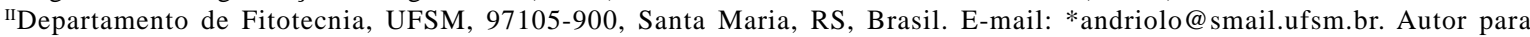
correspondência.

IIICurso de Agronomia, UFSM, Santa Maria, RS, Brasil. 
VASSEN, 2005). Decorre dessa teoria a idéia de uma concentração crítica nas plantas, necessária para maximizar o crescimento e a produtividade.

Os fatores que afetam o crescimento da planta também influenciam a absorção e a assimilação do N. Esse nutriente é absorvido e assimilado por meio de processos com alto custo energético, os quais são induzidos pela demanda da parte aérea em aminoácidos, proteínas e enzimas necessárias à síntese da matéria seca (SMART \& BLOOMM, 1993). Embora a célula vegetal apresente elevada capacidade de estocagem de $\mathrm{N}$ no vacúolo, essas quantidades são pequenas quando comparadas com a demanda para o crescimento (TAIZ \& ZEIGER, 2004). Isso significa que o manejo ideal do $\mathrm{N}$ é aquele capaz de ajustar as doses de adubação de acordo com a produção de biomatéria da cultura nas diferentes épocas e fases do seu ciclo de crescimento e desenvolvimento.

Dentre os métodos de diagnóstico da nutrição nitrogenada das culturas agrícolas, destacase a curva crítica de diluição (LEMAIRE et al., 1997). Essa curva representa a demanda ponderal de N, determinada pelo crescimento do compartimento metabólico e estrutural da planta (HARDWICK, 1987; CALOIN \& YU, 1984), estimada pela equação $\% \mathrm{~N}=$ a $\mathrm{MS}^{-\mathrm{b}}$, em que \%N representa a concentração crítica de $\mathrm{N}$ (Nc), MS a matéria seca da parte aérea em t ha ${ }^{-1}$ e a e b são coeficientes de ajuste do modelo. As quantidades de $\mathrm{N}$ extraídas pelas culturas com matéria seca aérea acumulada igual ou superior a $1 \mathrm{t} \mathrm{ha}^{-1}$ são estimadas pela equação transformada $\mathrm{KgN}=10 \mathrm{a} \mathrm{MS}{ }^{(1-b)}$. A Nc permite ainda interpretar os resultados da análise foliar, através de um índice de nutrição de nitrogênio (INN), obtido pelo quociente entre a Nc e a concentração da amostra (LEMAIRE et al., 1997).

Os coeficientes do modelo de diluição do $\mathrm{N}$ foram ajustados para culturas forrageiras (LEMAIRE \& SALETTE, 1984), trigo (JUSTES et al., 1994), tomateiro (RATTIN, 2000; TEI et al., 2002), batata (DUCHÈNNE et al., 1997; BÉLANGER et al., 2001; CHAMBENOIT et al., 2002; PAULA, 2005). No caso de cucurbitáceas, como a cultura do melão, esses coeficientes ainda não foram determinados, embora sejam culturas que requerem elevados níveis de adubação nitrogenada e atingem produtividades elevadas.

O objetivo deste trabalho foi determinar a curva crítica de diluição do $\mathrm{N}$ na cultura do melão cultivado em ambiente protegido.

\section{MATERIAL E MÉTODOS}

O experimento foi conduzido no Departamento de Fitotecnia da Universidade Federal de Santa Maria (UFSM), em um abrigo do tipo capela de $200 \mathrm{~m}^{2}$ coberto com polietileno aditivado anti-UV com $200 \mu \mathrm{m}$ de espessura. As plantas foram cultivadas no interior de sacolas de polietileno contendo $5 \mathrm{dm}^{3}$ de substrato comercial orgânico (PlantmaxPXT ${ }^{\circledR}$ ). As sacolas foram colocadas sobre camalhões cobertos com filme de polietileno opaco preto de $30 \mu \mathrm{m}$ de espessura, no espaçamento de $1 \mathrm{~m}$ entre fileiras e $0,33 \mathrm{~m}$ entre sacolas, correspondendo à densidade de 3,3 plantas $\mathrm{m}^{-2}$. As plantas foram conduzidas em fitas plásticas verticais, com uma única haste por planta, a qual foi despontada ao atingir a altura de $2 \mathrm{~m}$. A água e os nutrientes foram fornecidos através de um tubo gotejador no centro de cada sacola, com vazão equivalente a $1,3 \mathrm{~L} \mathrm{~h}{ }^{-1}$. O conjunto formado pelas sacolas e tubo gotejador foi coberto com filme opaco de polietileno dupla-face, para reduzir a evaporação e evitar a incidência direta dos raios solares sobre o substrato. O volume de água retida na capacidade máxima de retenção foi de $1.250 \mathrm{~mL}$ em cada sacola. A freqüência e o volume das fertirrigações foram estimados com base na demanda hídrica da cultura por unidade de radiação solar e de área foliar, a partir de resultados do consumo hídrico de hortaliças cultivadas no mesmo local em ambiente protegido (DALSASSO et al., 1997). Foi adotado um coeficiente de drenagem perdida de pelo menos $20 \%$ em cada fertirrigação.

Os tratamentos foram constituídos por cinco soluções nutritivas com concentrações de totais de nitrogênio ( $\left.\mathrm{NO}_{3}^{-} / \mathrm{NH}_{4}\right)$ de 8 (T1), 11 (T2), 14 (T3), 17 (T4) e $20 \mathrm{mmol} \mathrm{L}^{-1}$ (T5). Em T4 e T5, foi empregado

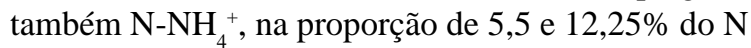
total, respectivamente, através do nitrato de amônio. Os demais nutrientes foram fornecidos nas mesmas concentrações para os cinco tratamentos, sendo de 0,9 de $\mathrm{H}_{2} \mathrm{PO}_{4}^{-} ; 2,25$ de $\mathrm{SO}_{4}^{-2} ; 10,0$ de $\mathrm{Ca}^{+2} ; 6,0$ de $\mathrm{K}^{+} \mathrm{e}$ 5,0mmol L-1 de $\mathrm{Mg}^{+2}$, e 0,42 de Mn; 0,26 de Zn; 0,05 de Cu; 0,50 de B; 0,04 de Mo e 4,82 $\mathrm{mg} \mathrm{L}^{-1}$ de Fe quelatizado em formulação pó. Cada uma das soluções nutritivas foi preparada em um reservatório separado, ao qual foi conectada uma motobomba. As cinco motobombas foram controladas por um único programador horário, de forma a fertirrigar concomitantemente todas as plantas na área experimental. O delineamento experimental foi o inteiramente casualizado, com quatro repetições de uma unidade experimental composta por uma fileira de 30 plantas.

No período entre 33 e 99 dias após o plantio (DAP), foram coletadas semanalmente quatro plantas de cada tratamento para determinação da matéria seca, sendo realizadas seis coletas. Imediatamente após as coletas, os limbos foliares, a haste, os pecíolos e os frutos foram separados, os quais foram secados em 
estufa de circulação forçada de ar, na temperatura de $60^{\circ} \mathrm{C}$, até matéria constante. A matéria seca de cada órgão foi moída em moinho tipo Willey e a concentração de $\mathrm{N}$ determinada pelo método Kjeldahl (TEDESCO et al., 1995).

A determinação dos pontos críticos para o ajuste da curva crítica de diluição do N (LEMAIRE et al., 1997) foi feita conforme descrito por JUSTES et al. (1994). Através deste método, as médias de acumulação da matéria seca das plantas que receberam as doses crescentes de $\mathrm{N}$ em cada coleta foram comparadas pelo teste $t$. Dois grupos de médias foram distinguidos, respectivamente com e sem efeito significativo das doses sobre o acúmulo de matéria seca. Duas equações lineares foram então ajustadas sobre esses pontos e o ponto crítico de diluição do $\mathrm{N}$ em cada coleta foi calculado pela resolução do sistema de equações. A curva crítica de diluição foi ajustada sobre os pontos críticos determinados nas seis coletas realizadas.

\section{RESULTADOS E DISCUSSÃO}

A concentração de $\mathrm{N}$ na planta inteira decresceu durante o crescimento e o desenvolvimento das plantas em todas as doses empregadas (Figura 1A). A concentração mais elevada de $N$ na primeira coleta de plantas efetuada aos 33 DAP foi observada nas plantas que receberam a dose $\mathrm{T} 5$, com valor ajustado de $6,2 \%$, representado pelo coeficiente $a$ do modelo (Tabela 1). Essa concentração decresceu com as doses até atingir o valor mais baixo de 4,1\% nas plantas que receberam a dose $\mathrm{T} 1$ no mesmo período. A intensidade da diluição foi mais elevada em T1, com coeficiente $b$ igual a $-0,93$ e decresceu com as doses até atingir o valor de -0,43 em T5. A curva crítica de diluição foi ajustada sobre os pontos críticos determinados pelo método de JUSTES et al. (1994), obtendo-se os valores de 5,16 e -0,63 para os coeficientes $a$ e $b$, respectivamente (Figura1B).

A diluição do $\mathrm{N}$ também foi observada nos órgãos da parte aérea das plantas, quando analisados separadamente (Figura 2). A concentração mais elevada atingiu 9\% e foi observada nas folhas das plantas de T5 aos 33 DAP. A concentração mais baixa atingiu 1,94\% e foi observada no caule das plantas de T1, aos 99 DAP. A maior intensidade de diluição foi observada no caule, com valor do coeficiente $b$ de-0,92 em T1 e a menor nos frutos de $\mathrm{T} 5$, com valor de $-0,07$ para esse coeficiente (Tabela 1 ).

A diluição do $\mathrm{N}$ observada na planta de melão demonstra que esse fenômeno também ocorre em hortaliças de crescimento indeterminado que produzem frutos, confirmando os resultados de RATTIN (2000) obtidos em plantas de tomateiro. Entretanto, constatou-se que a intensidade da diluição varia entre as espécies. As curvas críticas de diluição do $\mathrm{N}$ determinadas para outras culturas mostram valores do coeficiente $a$ e $b$ variando entre 3,4 e 5,5 e entre 0,29 e $-0,39$, respectivamente, para espécies anuais (LEMAIRE et al., 1997), de 4,2 e 0,27, para o tomateiro do tipo salada de crescimento indeterminado (RATTIN, 2000) e entre 3,6 e 5,2 e entre $-0,37$ e $-0,56$,
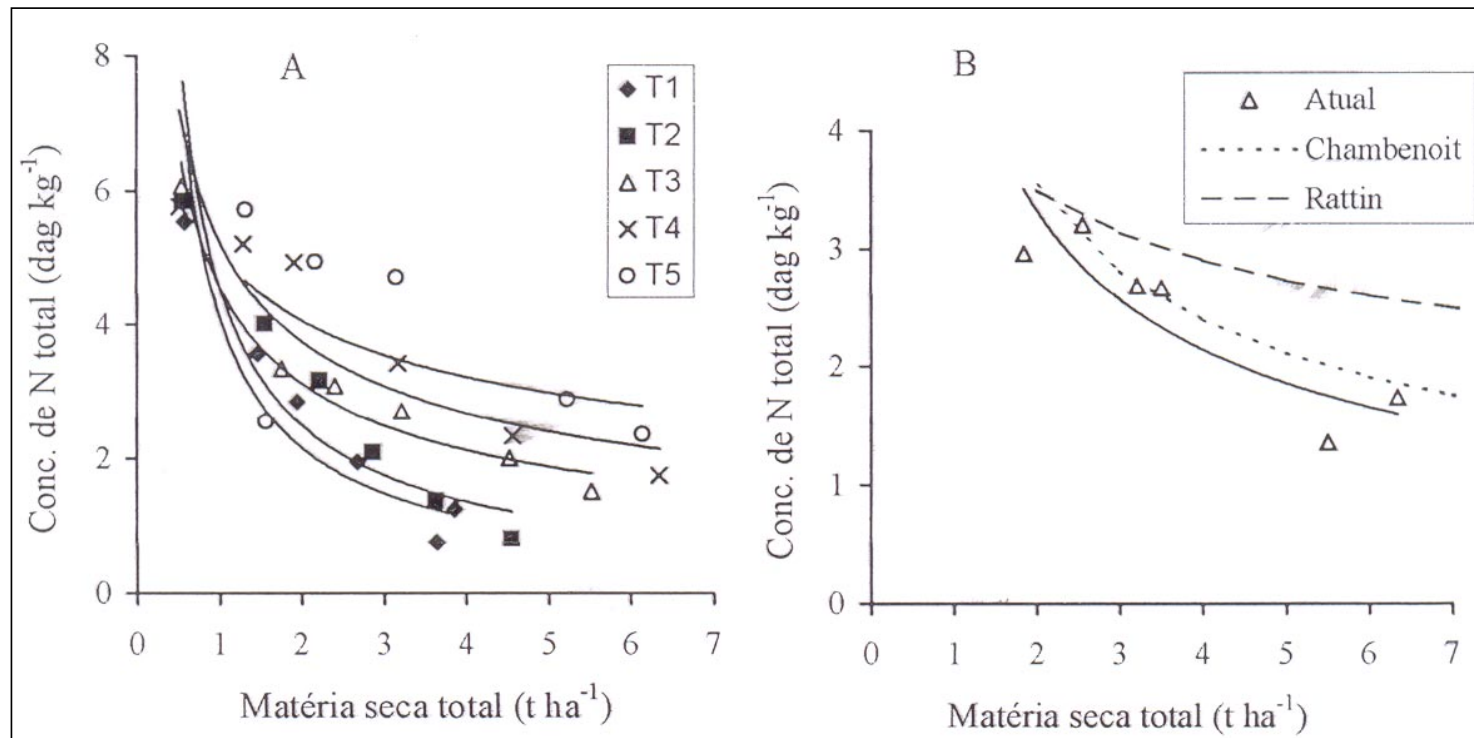

Figura 1 - Curvas de diluição do $\mathrm{N}$ de plantas de melão cultivadas com cinco concentrações de $\mathrm{N}$ na solução nutritiva (A). Curvas críticas de diluição (B): atual, ajustada com os resultados da Figura A (N, dag $\left.\mathrm{kg}^{-1}=5,16 \mathrm{MS}^{-0,63}, \mathrm{r}^{2}=0,76\right)$; da literatura para o tomateiro ( $\mathrm{N}$, dag $\mathrm{kg}^{-1}=4,22 \mathrm{MS}^{-0,27}$, RATTIN, 2000) e para a batata $\left(\mathrm{N}\right.$, dag $\mathrm{kg}^{-1}=5,21 \mathrm{MS}^{-0,56}$, CHAMBENOIT C.L. et al., 2002). Santa Maria, UFSM, 2005. 
Tabela 1 - Valores dos coeficientes $a, b$ e coeficientes de determinação das curvas ajustadas com o modelo de diluição do nitrogênio (N, dag $\mathrm{kg}^{-1}=\mathrm{a} \mathrm{MS}^{-\mathrm{b}}$ ) relativos à planta inteira e órgãos da planta, nas cinco doses fornecidas. Santa Maria, RS, UFSM, 2004.

\begin{tabular}{|c|c|c|c|c|}
\hline Material & Tratamento & Coeficiente $a$ & Coeficiente $b$ & $r^{2}$ \\
\hline \multirow[t]{5}{*}{ Planta inteira } & $\mathrm{T} 1$ & 4,1 & $-0,93$ & 0,99 \\
\hline & $\mathrm{T} 2$ & 4,6 & $-0,87$ & 0,92 \\
\hline & T3 & 4,6 & $-0,50$ & 0,88 \\
\hline & $\mathrm{T} 4$ & 5,2 & $-0,48$ & 0,88 \\
\hline & $\mathrm{T} 5$ & 6,2 & $-0,43$ & 0,88 \\
\hline \multirow[t]{5}{*}{ Folhas } & $\mathrm{T} 1$ & 4,5 & $-0,32$ & 0,88 \\
\hline & $\mathrm{T} 2$ & 3,8 & $-0,49$ & 0,63 \\
\hline & T3 & 3,5 & $-0,50$ & 0,56 \\
\hline & $\mathrm{T} 4$ & 5,0 & $-0,34$ & 0,85 \\
\hline & $\mathrm{T} 5$ & 5,9 & $-0,33$ & 0,93 \\
\hline \multirow[t]{5}{*}{ Caule } & $\mathrm{T} 1$ & 1,5 & $-0,92$ & 0,88 \\
\hline & $\mathrm{T} 2$ & 3,8 & $-0,49$ & 0,63 \\
\hline & $\mathrm{T} 3$ & 3,5 & $-0,50$ & 0,56 \\
\hline & $\mathrm{T} 4$ & 5,0 & $-0,34$ & 0,85 \\
\hline & $\mathrm{T} 5$ & 5,9 & $-0,33$ & 0,93 \\
\hline \multirow[t]{5}{*}{ Frutos } & $\mathrm{T} 1$ & 3,2 & $-0,34$ & 0,96 \\
\hline & $\mathrm{T} 2$ & 4,0 & $-0,14$ & 0,95 \\
\hline & $\mathrm{T} 3$ & 3,3 & $-0,39$ & 0,96 \\
\hline & $\mathrm{T} 4$ & 4,3 & $-0,42$ & 0,86 \\
\hline & $\mathrm{T} 5$ & 4,39 & $-0,07$ & 0,99 \\
\hline
\end{tabular}

respectivamente, para a cultura da batata (DUCHÈNE et al., 1997; BÉLANGER et al., 2001; CHAMBENOIT et al., 2002; PAULA, 2005).

O coeficiente $a$ indica a concentração de $\mathrm{N}$ nas fases jovens do desenvolvimento da cultura e a variação nos valores desse coeficiente encontrados na literatura pode ser atribuída tanto ao manejo da adubação da cultura como às condições ambientais. Quando doses elevadas de adubação nitrogenada são aplicadas no plantio, a concentração desse nutriente, nos tecidos da planta, aumenta nas fases iniciais do desenvolvimento da cultura porque a disponibilidade é elevada e a demanda pelo crescimento ainda é reduzida nessas fases. Quando a adubação é parcelada ao longo do período de produção da cultura, a concentração nessas fases iniciais é mais baixa porque a disponibilidade de N é menor. A concentração mantémse elevada também sob condições ambientais que favorecem o crescimento vegetativo da cultura, retardando a emissão das estruturas de reprodução e estocagem, pois a diluição do $\mathrm{N}$ é conseqüência das diferenças entre as dinâmicas de crescimento do compartimento vegetativo e de estocagem.

$O$ coeficiente $b$ indica a intensidade da diluição e suas variações são atribuídas principalmente à espécie e ao manejo da cultura. Os valores desse coeficiente são menores (mais negativos) em espécies de crescimento determinado, nas quais o crescimento da parte vegetativa cessa ao ter início a fase de crescimento dos órgãos de acumulação e reserva. Essa hipótese explica os valores na faixa entre - 0,30 e $-0,40$ em culturas anuais de crescimento determinado e de 0,27 no tomateiro indeterminado. Porém, para os dados atuais da cultura do melão, o valor foi de -0,63. Esse fato pode ser atribuído tanto à condução da cultura como às relações de partição da matéria seca que a caracterizam. No cultivo do melão em ambiente protegido, são empregadas elevadas densidades de plantas, as quais são conduzidas com fitas plásticas verticais, com remoção sistemática das ramificações axilares e poda apical ao atingir a altura máxima permitida pela estrutura de sustentação. Essa prática tem como conseqüência a limitação precoce do crescimento da área foliar, envelhecendo o aparato vegetativo da planta, e, portanto, aproximando-se da situação de uma cultura de crescimento determinado. Com relação à partição da matéria seca, a planta do melão caracterizase por forte competição entre o crescimento da parte vegetativa e dos frutos (CTIFL, 1998), a qual intensificaria a remobilização de assimilados e nutrientes da área foliar remanescente. Esse processo também se assemelha ao que ocorre nas culturas de crescimento determinado. As variações entre os valores do coeficiente $b$ determinado e aqueles encontrados na literatura explicam-se, portanto, tanto pelo hábito de crescimento como pela forma de condução da cultura do melão.

Ciência Rural, v.38, n.2, mar-abr, 2008. 
As curvas de diluição nos órgãos da parte aérea (Figura 2) indicam variações relacionadas tanto à idade fisiológica da cultura quanto à disponibilidade
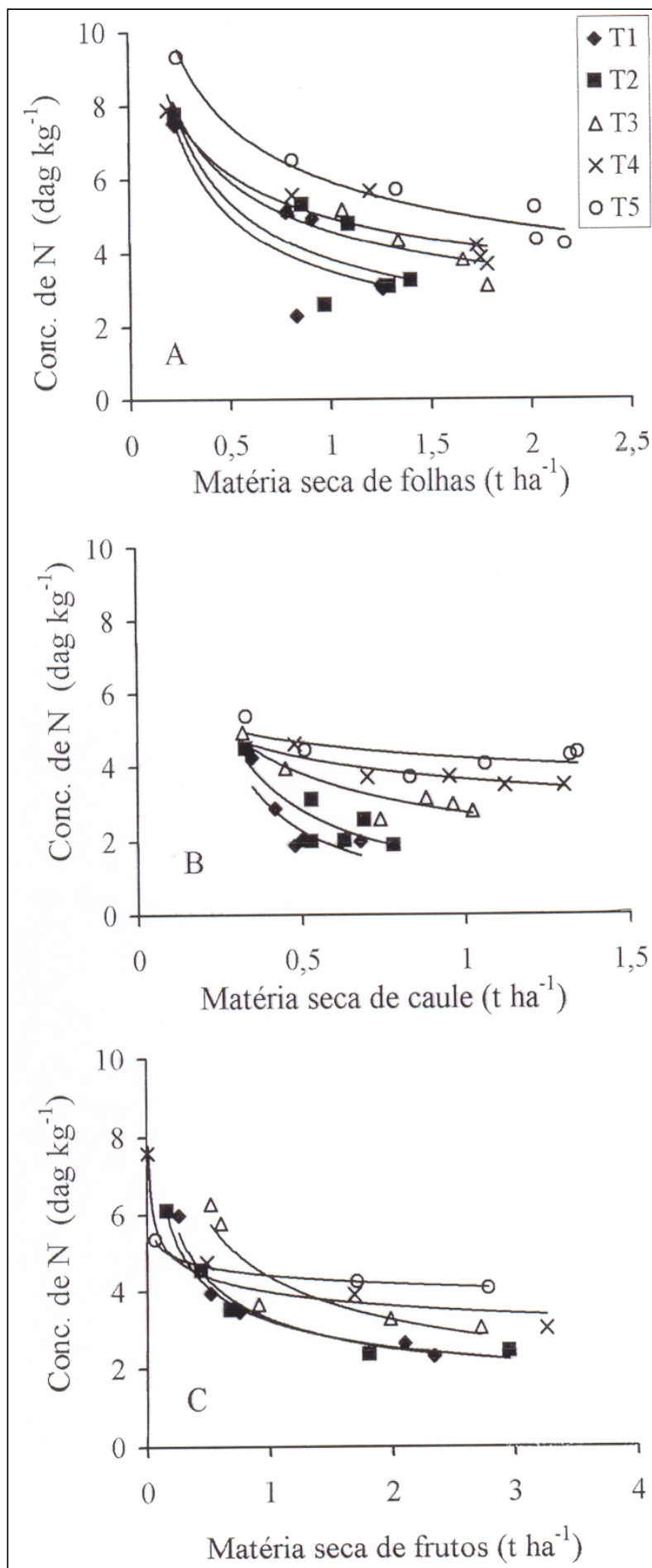

Figura 2 - Curvas críticas de diluição do $\mathrm{N}$ nas folhas (A), hastes (B) e frutos (C) no decorrer do período de crescimento e desenvolvimento de plantas de melão. Santa Maria, UFSM, 2005 de N. Isso significa que a interpretação dos resultados da análise de tecidos pode conduzir a erros quando esses fatores não são considerados. Essas variações podem explicar a ampla gama de valores indicada na literatura como adequada para essa cultura. TRANI \& RAIJ (1997) indicam teores médios de nitrogênio para pecíolo e limbo foliar de 2,7 e 4,1dag $\mathrm{kg}^{-1}$, respectivamente, com uma faixa ideal entre 2,5 e 5,0dag $\mathrm{kg}^{-1}$. Do ponto de vista do manejo da adubação da cultura, somente as curvas críticas de diluição em nível da planta inteira devem ser consideradas porque representam a média ponderada das concentrações de $\mathrm{N}$ e das frações da matéria seca total. Por essas razões, a definição de um tecido foliar amostral com representatividade em nível da planta inteira no decorrer de todo o ciclo de crescimento e desenvolvimento deve ser objeto de determinação em experimentos futuros com essa cultura, conforme estudo realizado por RATTIN et al. (2002) sobre o tomateiro. No caso das hastes e pecíolos (Figura 2B), aportantoportantoportantos curvas de diluição sugerem que esses órgãos podem funcionar como sítios de estocagem temporária de $\mathrm{N}$, o que dificultaria a interpretação dos resultados das análises de laboratório. Essa observação deixa dúvidas sobre a conveniência de empregar esses tecidos em análises para o diagnóstico nutricional. No caso dos frutos, os teores de $\mathrm{N}$ são elevados apenas nas fases iniciais do seu ciclo de crescimento e desenvolvimento e mostram pequena variação após ultrapassar o limite de $1 \mathrm{t} \mathrm{ha}^{-1}$ (Figura 2C).

A produtividade de frutos variou entre 39,6 e 62,2t ha ${ }^{-1}$ nos tratamentos T1 a T5, respectivamente. A maior fração da matéria seca total alocada para os frutos foi de 0,73 em T3, que foi o tratamento mais próximo da curva crítica. Esse resultado confirma que os frutos são os órgãos que determinam de forma ponderal as necessidades de $\mathrm{N}$ da cultura. Para o manejo da adubação nitrogenada, a curva crítica de diluição determinada pode ser empregada em duas fases distintas do ciclo. A primeira é no momento do plantio, para estimar as quantidades extraídas pela cultura, levando em conta a densidade da lavoura e a produtividade de frutos a ser atingida. O cálculo pode ser feito empregando-se o modelo $\mathrm{Kg} \mathrm{ha}^{-1} \mathrm{~N}=51,6 \mathrm{MS}^{(1-0,63)}$. As estimativas para produtividades diferentes da atual poderão ser proporcionalmente ajustadas, com base naquela fração.

Na segunda fase, as quantidades totais de $\mathrm{N}$ estimadas durante o período de crescimento

Ciência Rural, v.38, n.2, mar-abr, 2008. 
e desenvolvimento da cultura podem ser parceladas de acordo com a acumulação de matéria seca (MS). Para tal, a MS de lavouras com densidade e condução similares àquelas do presente experimento poderá ser estimada pela expressão MS (ton ha $\left.{ }^{-1}\right)=$ 0,0005DAP ${ }^{2}+0,1314 \mathrm{DAP}-3,40$. Essa equação foi ajustada a partir dos resultados de crescimento do atual experimento, sendo a variável DAP expressa em dias decorridos após o plantio. Nessa fase, as análises de tecidos no laboratório poderão servir de elementos de diagnóstico do estado nutricional da lavoura, inferindose as necessidades de adubação com base nos teores indicados pela curva crítica de diluição, para a respectiva fase indicada pela matéria seca acumulada.

\section{CONCLUSÕES}

Os valores ajustados dos coeficientes $a$ e $b$ determinam a curva crítica de diluição $\mathrm{N}$ (dag $\left.\mathrm{kg}^{-1}\right)=5,16 \mathrm{MS}^{-0,63}$ e a curva crítica de extração $\mathrm{N}\left(\mathrm{Kg} \mathrm{ha}^{-1}\right)=51,6 \mathrm{MS}^{(1-0,63)}$. A expressão MS (ton ha ${ }^{-1}$ ) $=-0,0005$ DAP $^{2}+0,1314$ DAP-3,40 pode ser empregada para estimar a acumulação de matéria seca durante o período de crescimento e desenvolvimento de primavera-verão, com base nos dias decorridos desde o plantio (DAP).

\section{AGRADECIMENTOS}

Ao Conselho Nacional de Desenvolvimento Científico e Tecnológico (CNPq), pela Bolsa de Produtividade em Pesquisa ao professor Jerônimo Luiz Andriolo e de Iniciação Científica (IC) aos acadêmicos de Agronomia Rodrigo dos Santos Godoi e Djeimi Isabel Janisch.

\section{REFERÊNCIAS}

BÉLANGER, G.W. et al. Critical nitrogen curve and citrogen nutrition index for potato in eastern Canada. American Journal of Potato Research, v.78, p.355-364, 2001.

CALOIN, M; YU, O. Analysis of the time course of change in nitrogen content in Dactilys glomerata L. using a model of plant growth. Annals of Botany, v.54, p.64-76, 1984.

CHAMBENOIT, C.L. et al. Fertilisacion azotée de la pomme de terre. Paris: INRA, 2002. 128p.

CTIFL. Centre Technique Interprofessionel des Fruits e des Légumes. Le melon. Paris: CTIFL, 1998. 165p.

DALSASSO, L.C.M. et al. Consumo de água do tomateiro tipo salada em estufa plástica. Revista Brasileira de Agrometeorologia, v.5, n.1, p.61-67, 1997.
DUCHÈnNE, T. et al. Potatoes. In : LEMAIRE, G. (Ed.). Diagnosis of the nitrogen status in crops. Berlin: SpringerVerlag, 1997. p.119-130.

HARDWICK, R.C. The nitrogen content of plants and the slfthining rule of plant ecology: a test of the core-skin hypothesis. Annals of Botany, v.60, p.139-446, 1987.

INGESTAD, T.; ÅGREN, G.I. Theories and methods on plant nutrition and growth. Physiologia Plantarum, v.84, p.177184, 1992.

JUSTES, E. et al. Determination of a critical nitrogen dilution curve for winter wheat crops. Annals of Botany, v.74, p.397404, 1994.

LEMAIRE, G. et al. Dynamics of $\mathrm{N}$ uptake and $\mathrm{N}$ distribution in plant canopies. Use of crop $\mathrm{N}$ status index in crop modelling. In : LEMAIRE, G. (Ed.). Diagnostic procedures for crop $\mathbf{N}$ management. Paris: INRA, 1997. p.16-29.

LEMAIRE, G.; SALETTE, J. Croissance estivale en matière séche de peuplements de fétuque élevée (Festuca arundinacea Schreb) et de dactyle (Dactylis glomerata L.) dans l'ouest de la France. I. Etude em conditions de nutrition azotée ect d'alimentation hydrique non limitantes. Agronomie, v.7, n.6, p. 381-389, 1984.

PAULA, A.L. de. Acúmulo de matéria seca e nitrogênio durante o crescimento e desenvolvimento da cultura da batata. 2005. 23f. Dissertação (Mestrado em Agronomia) Programa de Pós-graduação em Agronomia, Universidade Federal de Santa Maria.

RATTIN, J.E. Acumulação de matéria seca e teores de nitrogênio na planta do tomateiro cultivado em substrato sob cinco doses de nutrientes. 2000. 71f. Dissertação (Mestrado em Agronomia) - Programa de Pós-graduação em Agronomia, Universidade Federal de Santa Maria.

SMART,D.R; BLOOM, M.J. Relationships between the kinetics of $\mathrm{NH}+4$ and NO-3 absorption and growth in the cultivated tomato (Lycopersicon esculentum cv. T-5). Plant, Cell and Environment, v.16, p.259-267, 1993.

TAIZ, L.; ZEIGER, E. Fisiologia vegetal. 3.ed. Porto Alegre: Artmed, 2004. 719p.

TEDESCO, M.J. et al. Análise de solo, plantas e outros materiais. Porto Alegre: UFRGS, 1995. 173p.

TEI, F. et al. Critical nitrogen concentration in processing tomato. European Journal of Agronomy, v.18, p.45-55, 2002.

TRANI, P.E.; RAIJ, B.V. Hortaliças. In: RAIJ, B.V. et al. Recomendações de adubação e calagem para o Estado de São Paulo. São Paulo: Fundação IAC, 1996. p.155-185.

VERKROOST, A.W.M.; WASSEN, M.J. A simple model for nitrogen-limited plant growth and nitrogen allocation. Annals of Botany, v.96, p.871-876, 2005. 